Check for updates

Cite this: RSC Adv., 2018, 8, 35917

Received 26th August 2018

Accepted 16th October 2018

DOI: $10.1039 / c 8 r a 07123 c$

rsc.li/rsc-advances

\section{Preparation and evaluation of poly(ester-urethane) urea/gelatin nanofibers based on different crosslinking strategies for potential applications in vascular tissue engineering}

\author{
Yao Wang, $\uparrow^{\mathrm{a}}$ Tonghe Zhu, $\dagger^{\mathrm{b}}$ Haizhu Kuang, $\dagger^{\mathrm{b}}$ Xiaoning Sun, ${ }^{\mathrm{a}}$ Jingjing Zhu, ${ }^{\mathrm{b}}$ Yu Shi, \\ Chunsheng Wang, ${ }^{a}$ Xiumei Mo, (D) *b Shuyang Lu (D) *a and Tao Hong ${ }^{* a}$
}

\begin{abstract}
Due to the brittleness of gelatin, the resulting absence of mechanical performance restricts its applications in vascular tissue engineering. In this research, the fabrication of poly(ester-urethane) urea/gelatin (PU75) nanofibers via an electrospinning technique, followed by different crosslinking methods, resulted in the improvement of its mechanical properties. Poly(ester urethane) urea (PEUU) nanofibrous scaffolds and PU75-based nanofibrous scaffolds were characterized using scanning electron microscopy (SEM), attenuated total reflection Fourier transform infrared (ATR-FTIR) spectroscopy, wide-angle X-ray diffraction (WAXRD), a mechanical properties test, a cytocompatibility assay, a hemolysis assay, and a histological analysis. Water contact angle (WCA) tests confirmed that the PU75-GA (PU75 nanofibers crosslinked with glutaraldehyde vapor) nanofibrous scaffold surfaces became more hydrophilic compared with other crosslinked nanofibrous scaffolds. The results show that the PU75-GA nanofibrous scaffold exhibited a combination of excellent mechanical properties, suitable pore diameters, hydrophilic properties, good cytocompatibility, and reliable hemocompatibility. Overall, PU75-GA nanofibers may be a potential scaffold for artificial blood vessel construction.
\end{abstract}

\section{Introduction}

For vascular tissue engineering scaffolds, prominent biocompatibility and mechanical properties are extremely important. ${ }^{1}$ In recent years, electrospun nanofibers have been widely used in vascular tissue engineering thanks to their high porosity and surface-to-volume ratio. ${ }^{2}$ Attempts to mimic the extracellular matrix (ECM) have resulted in the development of a nanofiberbased scaffold with good hydrophilic properties. ${ }^{3}$ Gelatin, a polydisperse mixture of peptides and proteins, has the advantage of a better inherent biocompatibility than synthetic polymers and a greater possibility for modification. ${ }^{4-6}$ However, a pure gelatin scaffold is very fragile following various processing methods, such as self-assembly, phase separation, wetspinning, dry-spinning, and electrospinning, among others. ${ }^{7-9}$

Biodegradable polyurethanes represent attractive potential candidate biomaterials for use in tissue-engineered vascular scaffold construction due to their elasticity and tunable

\footnotetext{
${ }^{a}$ Department of Cardiovascular Surgery, Zhongshan Hospital, Fudan University, The Shanghai Institute of Cardiovascular Diseases, Shanghai 200032, People's Republic of China. E-mail: hong.tao@zs-hospital.sh.cn; lu.shuyang@zs-hospital.sh.cn ${ }^{b}$ State Key Laboratory for Modification of Chemical Fibers and Polymer Materials, College of Chemistry, Chemical Engineering and Biotechnology, Donghua University, Shanghai 201620, People's Republic of China. E-mail: xmm@dhu.edu.cn

$\dagger$ These authors contributed equally to this work.
}

mechanical and degradation properties. ${ }^{10}$ Poly(ester-urethane) urea (PEUU) is one critical member of the biodegradable polyurethane family due to its cytocompatibility, non-toxic degradation products, and ability to be processed into scaffolds via various techniques. ${ }^{11-13}$ This material, with different types of soft and hard segments, has been used for tissue-engineering scaffolds and is expected to be an excellent alternative due to its tunable mechanical properties, biodegradability, biocompatibility, and ability to be processed into scaffolds via various techniques. ${ }^{\mathbf{1 4 , 1 5}}$

Gelatin has ubiquitously been electrospun in combination with synthetic polymers, mostly by blending ${ }^{7}$ or coating. ${ }^{16}$ Very few reports show and evaluate the utilization of different crosslinking methods to achieve excellent biocompatibility and mechanical properties of PEUU/gelatin nanofibers. With our earlier work, a series of PEUU-based elastomers was synthesized, with nanofibers developed via electrospinning for biomedical applications, including vascular tissue engineering scaffolds, ${ }^{17}$ wound dressing, ${ }^{15}$ and drug delivery systems. ${ }^{18}$ However, the slow degradation time and hydrophobic surfaces of the nanofibers may not be appropriate for fabricating vascular tissue engineering materials. For gelatin, a relatively fast degradation time is detrimental to the material retaining its mechanical strength, while a highly hydrophilic surface may experience rapid dissolution during cell culturing or 
implantation. ${ }^{19}$ Therefore, to overcome their respective defects and capitalize on their respective advantages, the crosslinked PEUU/gelatin hybrid nanofibrous structure could be acquired from electrospinning for successful use in tissue engineering applications. This study focuses on the preparation and preliminary study of PEUU/gelatin composite nanofibers based on different crosslinking strategies to overcome the brittleness of gelatin and the poor biocompatibility of PEUU. The chemical structure, fiber morphology, mechanical properties, surface wettability, pore size, porosity, cell viability and morphology, hemocompatibility, and subcutaneous implantation of the composite nanofibrous scaffolds were investigated.

\section{Materials and methods}

\subsection{Materials}

PEUU samples were synthesized from 1,6-hexamethylene dissocyanate (HDI) and polycaprolactone diol (HO-PCL-OH) with butanediamine chain extensions, as shown in our previous research. ${ }^{15,17}$ The PEUU was estimated to have a weight-average molecular weight of $1.16 \times 10^{5}$ and a number-average molecular weight of $5.4 \times 10^{4}$. Gelatin (Gel, type B from porcine skin) was obtained from Sigma-Aldrich Chemical Reagent Co., Ltd (Saint Louis, USA). 1,1,1,3,3,3-Hexafluoro-2-propanol (HFIP) was acquired from Shanghai Darui Fine Chemical Co., Ltd. (Shanghai, China). The mouse fibroblast (L929) cells for in vitro analysis were obtained from the Shanghai Institute of Biochemistry and Cell Biology (SIBCB, CAS, China). All culture media and reagents, unless otherwise stated, were purchased from Invitrogen and Sigma-Aldrich (Saint Louis, USA).

\subsection{Fabrication of electrospun nanofibers}

The PU75 nanofibrous scaffold (referred to as PU75) was prepared using an electrospinning process. ${ }^{15}$ Succinctly, blends of PEUU/gelatin with weight ratios of $75 / 25$ were dissolved in HFIP to form $12 \% \mathrm{w} / \mathrm{v}$ solutions. Then, the solutions were stirred for $24 \mathrm{~h}$. The polymer solution was fed at $1.0 \mathrm{~mL} \mathrm{~h}^{-1}$ from a capillary tube charged at $-10 \mathrm{kV}$, located perpendicularly $14 \mathrm{~cm}$ from the target thin aluminum foil or SEA $316 \mathrm{~L}$ grade stainless steel bar. All the electrospinning processes were carried out at approximately $25{ }^{\circ} \mathrm{C}$ and $38 \% \pm 2 \%$ relative humidity. This preparation of a PEUU nanofibrous scaffold can be found in our previous research. ${ }^{17}$

\subsection{Crosslinking of electrospun PU75 nanofibers}

The dehydrothermal-treated scaffold (known as PU75-DT) was prepared using the following 2 steps: ${ }^{20}$ (1) electrospun PU75 nanofibrous membranes were cut into small pieces $(5 \mathrm{~cm} \times 3$ $\mathrm{cm})$. Nanofibrous tubular scaffold samples with inner diameters of $1.8 \mathrm{~mm}$ were cut to lengths of $1 \mathrm{~cm}$; (2) the strips of nanofibrous membrane or nanofibrous tubular scaffolds were heat-treated at $190{ }^{\circ} \mathrm{C}$ for $3 \mathrm{~min}$ in air. For comparison, PU75 nanofibrous membranes and nanofibrous tubular scaffolds crosslinked with glutaraldehyde vapor (named PU75-GA) were also prepared. Crosslinking was carried out by placing samples into $10 \mathrm{~mL}$ of a $25 \%$ glutaraldehyde solution (aqueous). ${ }^{1521}$ The nanofibrous membranes or nanofibrous tubular scaffolds, along with beakers, were kept inside a sealed desiccator and allowed to crosslink with glutaraldehyde vapor for $3 \mathrm{~h}$ at room temperature. The crosslinked nanofibrous membranes and nanofibrous tubular scaffolds were then washed in a $5 \%$ glutamic acid solution (1.8\% hydrochloric acid as a solvent) and doubly-distilled water, respectively, then dried under reduced pressure prior to use. 1-Ethyl-3-(3'-dimethylaminopropyl)carbodiimide/ $N$-hydroxysuccinimide (EDC/NHS)-crosslinked nanofibrous membranes and nanofibrous tubular scaffolds (named PU75-E/N) were prepared by the following steps. ${ }^{22,23}$ EDC $(0.35 \mathrm{~g})$ and NHS (0.15 g) were dissolved in $100 \mathrm{~mL}$ of $95 \%$ ethanol and stirred for $30 \mathrm{~min}$ to get a homogeneous EDC/NHScrosslinked solution. The $5 \mathrm{~cm} \times 3 \mathrm{~cm}$ strips of nanofibrous membrane and the nanofibrous tubular scaffolds with lengths of $1 \mathrm{~cm}$ were dispersed in the EDC/NHS-crosslinked solution for $3 \mathrm{~h}$, then washed in doubly-distilled water. All the samples were freeze-dried for $48 \mathrm{~h}$ before testing.

\subsection{Characterization and performance testing of nanofibrous scaffolds}

The structure of the electrospun nanofibers were investigated using SEM ${ }^{24}$ (Phenom, Phenom XL, Netherlands) at an accelerated voltage of $10 \mathrm{kV}$ after sputter-coating with gold. The pore sizes of all samples $(30 \mathrm{~mm} \times 30 \mathrm{~mm}, n=3)$ were measured by a CFP-1100-AI capillary flow porometer (PMI Porous Materials Int.) both before and after cross-linking. Porosity was calculated by: $\varepsilon=\left(1-\rho / \rho_{0}\right) \times 100 \%$, where $\rho$ is the density of nanofibrous membranes and $\rho_{0}$ is the density of cast film (the $\rho_{0}$ of PEUU, PU75, PU75-DT, PU75-GA, PU75-E/N being $1.23 \mathrm{~g} \mathrm{~cm}^{-3}$, $1.19 \mathrm{~g} \mathrm{~cm}^{-3}, 1.20 \mathrm{~g} \mathrm{~cm}^{-3}, 1.21 \mathrm{~g} \mathrm{~cm}^{-3}, 1.21 \mathrm{~g} \mathrm{~cm}^{-3}$, respectively). The water contact angle was measured 3 times for each sample using a contact angle device (OCA40, Data-physics, Germany) at room temperature.

Attenuated total reflection Fourier transform infrared (ATRFTIR) spectra were obtained in absorption mode at $2 \mathrm{~cm}^{-1}$ intervals in the range of $800-4000 \mathrm{~cm}^{-1}$ using an ATR-FTIR spectrophotometer (Avatar380, USA). Wide-angle X-ray diffraction (WAXRD) curves were obtained on a D/max-2550 PC X-ray diffractometer (Rigaku Co., Tokyo, Japan) within the scanning region of $2 \theta\left(5-60^{\circ}\right)$, with $\mathrm{Cu} \mathrm{K} \alpha$ radiation $(l=1.5418 \AA)$ at $40 \mathrm{kV}$ and $40 \mathrm{~mA}$.

The mechanical properties of the nanofibrous membranes were tested in both dry and wet states with a material testing machine (H5K-S, Hounsfield, UK) at a crosshead speed of 10 $\mathrm{mm} \min ^{-1}$ under a load of $10 \mathrm{~N}$, according to ASTM D638-98 ( $n$ $=5)$. For wet states, the strips were immersed in PBS $\left(37^{\circ} \mathrm{C}\right)$ for $48 \mathrm{~h}$ and tested immediately following the removal from the PBS at room temperature. The stress and strain data were calculated using eqn (1) and eqn (2): ${ }^{\mathbf{1 4 , 1 5 , 1 7 , 1 8}}$

$$
\begin{aligned}
\sigma(\mathrm{MPa}) & =\frac{P(\mathrm{~N})}{w(\mathrm{~mm}) \times d(\mathrm{~mm})} \\
\varepsilon & =\frac{l}{l_{0}} \times 100 \%
\end{aligned}
$$


where $\sigma, \varepsilon, P, w, d, l$, and $l_{0}$ stand for stress, strain, load to failure, membrane width, membrane thickness, extension length, and gauge length (original length), respectively. Tensile strength, elongation at break, and Young's modulus values were obtained from the strain-stress curves. Cyclic stretch testing in dry states was conducted by stretching the strips $(3 \times 20 \mathrm{~mm}, n$ $=5)$ to strain at low-deformation $(30 \%)$ and high-deformation $(100 \%)$ and then releasing back to $0 \%$ strain. This measurement was repeated for 10 cycles at a fixed rate of $10 \mathrm{~mm} \mathrm{~min}^{-1}$ by an Instron 5969 testing system with a $200 \mathrm{~N}$ loaded sensor.

All samples were prepared at the same size $(20 \mathrm{~mm} \times 10$ $\mathrm{mm}$, about $0.3 \mathrm{~mm}$ in thickness). The suture retention strength was tested by an Instron5969 mechanical tester (Instron Corporation, USA). Before testing, a loop of a 5-0 polypropylene sutures (Ethilon, Ethicon, Inc., USA) was placed $2 \mathrm{~mm}$ from the edge of one end of the sample, with the other end of the sample clamped to one arm of the machine. Then, the suture was clamped to the other arm and stretched at a constant rate of 10 $\mathrm{mm} \min ^{-1}$ until failure. ${ }^{25}$ The maximum suture retention strength was recorded. Five specimens were tested for each sample and their results averaged.

Burst pressure testing was performed by a balloon pump pressure method (pressure syringe, All well Medical Inc., USA). PBS was filled into a $20 \mathrm{~mL}$ syringe and inserted at the end of the sample tube, while the other end was also firmly secured and sealed with 5-0 sutures ligature to prevent leakage. The pressure was increased by $300 \mathrm{mmHg} \mathrm{s}^{-1}$ until either failure or leakage occurred. The burst pressure was recorded as the maximum pressure prior to construct failure or leakage.

\subsection{Cell compatibility experiments and hemolysis test in} vitro of the prepared nanofibers specimen

For cell proliferation tests, L929 cells were seeded with a density of $5 \times 10^{3}$ cells per well. A Cell Counting Kit (CCK-8) assay and SEM (Phenom, Phenom XL, Netherlands) observations were employed to evaluate the viability and morphology, respectively, of the proliferated L929 cells cultured onto different nanofibrous membranes ( $n=4$ for each group), according to the manufacturer's protocol.

The detailed experimental procedures of the hemolysis test in vitro were followed according to the protocol of GB/T 16886.42003 of China. The experimental sample set consisted of a PEUU nanofibrous membrane, a PU75 nanofibrous membrane, a PU75-DT nanofibrous membrane, a PU75-GA nanofibrous membrane, and a PU75-E/N nanofibrous membrane $(3 \mathrm{~cm} \times 1 \mathrm{~cm})$. Milli-Q water served as a positive control and $0.9 \%$ normal saline $(0.9 \%$ NS) acted as a negative control.

\subsection{Subcutaneous implantation and histological analysis}

All animal experiments were performed in compliance with the protocols approved by the Institutional Animal Care and Use Committees (IACUC) guidelines of Fudan University. Nanofibrous tubes (with inner diameters of $2 \mathrm{~mm}$ and lengths of $5 \mathrm{~mm}$ ) were sterilized with ethylene oxide and then implanted symmetrically in 2 sides of the same mouse's back. After 4 weeks and 8 weeks, the nanofibrous tubes were removed from the backs of the mice. Then, the implants with

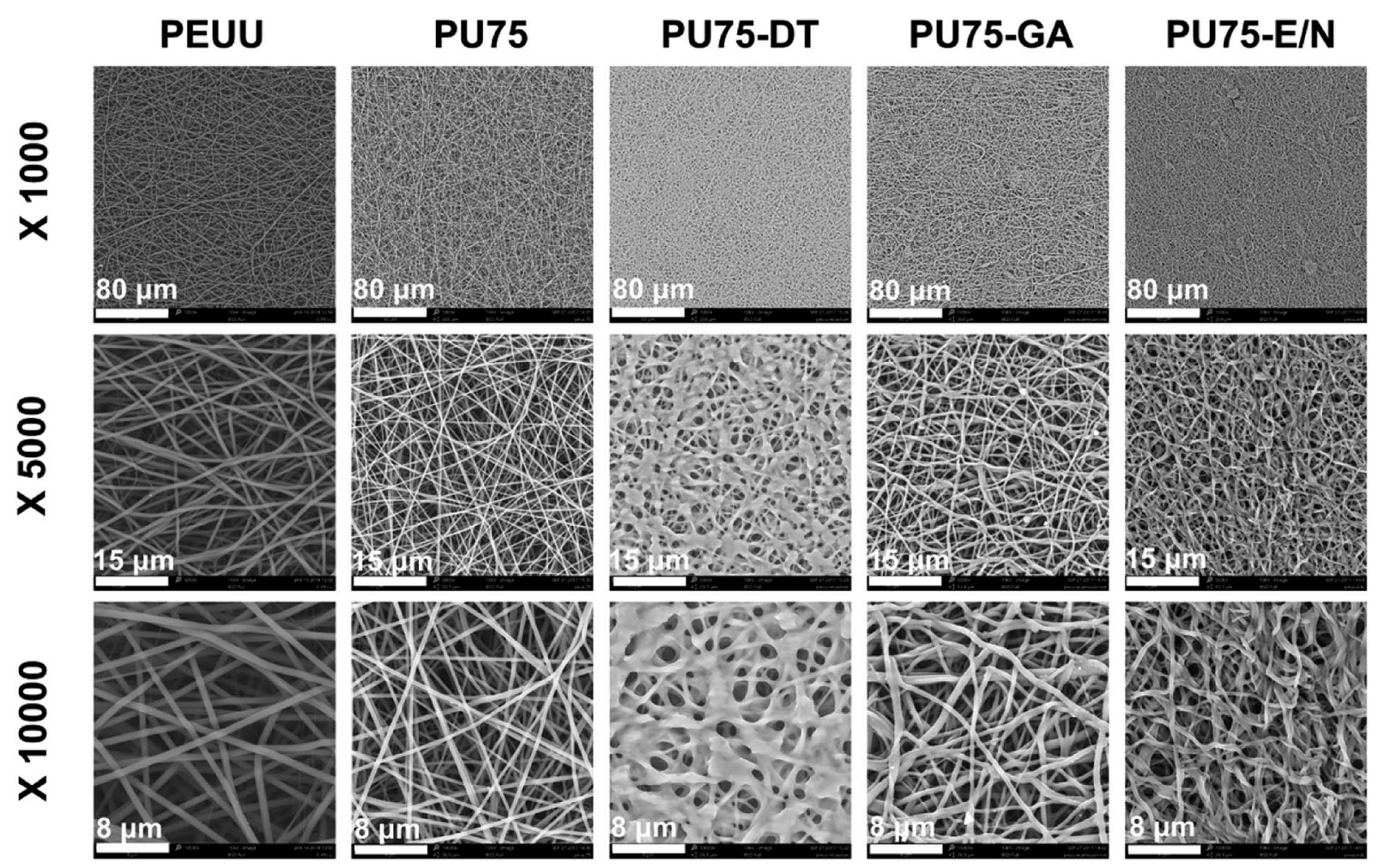

Fig. 1 SEM micrographs of the PEUU nanofibrous membrane, PU75 nanofibrous membrane, PU75-DT nanofibrous membrane, PU75-GA nanofibrous membrane, and PU75-E/N nanofibrous membrane and magnified 1000, 5000, and 10000 times, respectively. 
Table 1 Water contact angles, mean pore diameters, and porosity of PEUU, PU75, PU75-DT, PU75-GA, and PU75-E/N nanofibrous membranes

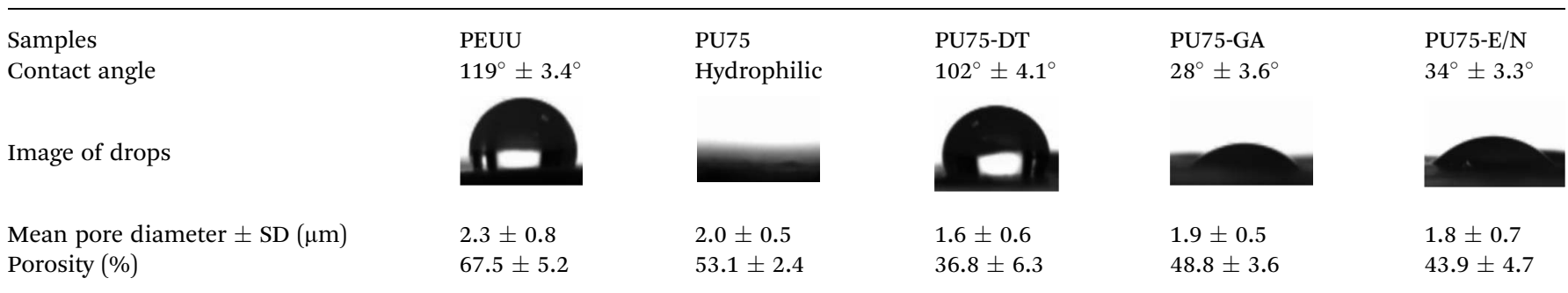

tissues were fixed with $4 \%$ paraformaldehyde for $48 \mathrm{~h}$ and dehydrated with a grade series of ethanol. ${ }^{26}$ The sections were stained with hematoxylin, eosin, and Masson's trichrome for analysis.

\subsection{Statistical analysis}

The data were analyzed using one-way analysis of variance (ANOVA), followed by Tukey's test for the evaluation of specific differences with Origin Pro 8.0 (Origin lab Inc., USA).

\section{Results and discussion}

\subsection{The morphology and hydrophilicity of nanofibers}

SEM morphological images of the PEUU nanofibrous membrane, PU75 nanofibrous membrane, PU75-DT nanofibrous membrane, PU75-GA nanofibrous membrane, and PU75-E/N nanofibrous membrane are presented in Fig. 1 . The results show that the obtained PEUU nanofibrous membrane and PU75 nanofibrous membrane present uniform, random (a)
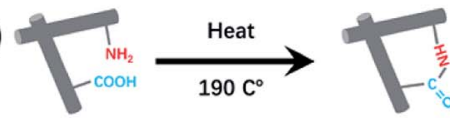

$+\mathrm{H}_{2} \mathrm{O}$

PU75 molecular chain

PU75-DT molecular chain (b)

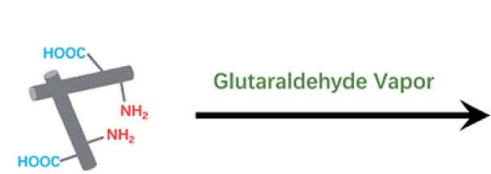

PU75 molecular chain

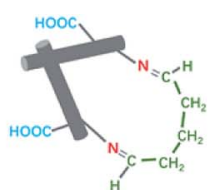

PU75-GA molecular chain

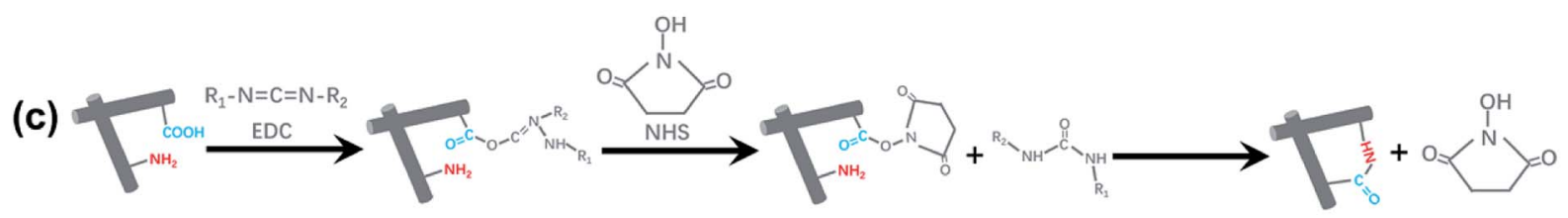

PU75 molecular chain

PU75-E/N molecular chain

Fig. 2 The formation mechanisms of crosslinking PU75: (a) crosslinking with dehydrothermal treatment; (b) crosslinking with glutaraldehyde vapor; (c) crosslinking with EDC/NHS solution.


Fig. 3 (a) ATR-FTIR spectra, (b) WAXRD pattern of gelatin nanofibers, PEUU nanofibers, PU75 nanofibers, PU75-DT nanofibers, PU75-GA nanofibers, and PU75-E/N nanofibers. 


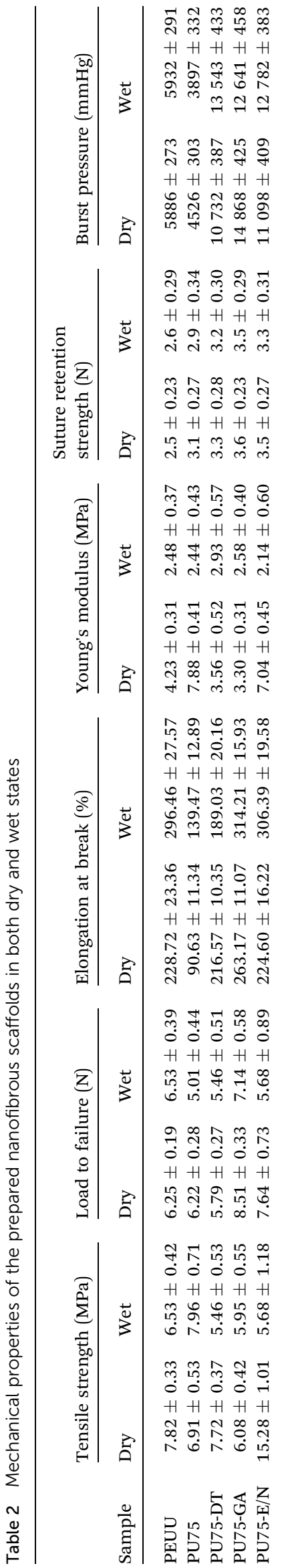

deposition. The diameters of the PU75 nanofibers are smaller than those of the PEUU nanofibers, presumably due to the increase of the solution conductivity or the solution viscosity caused by the introduction of gelatin in the electrospinning solution. The crosslinked nanofibrous membranes seem to significantly alter the uniform and smooth fibrous morphology when compared with PU75 nanofibrous membranes. In particular, the glutaraldehyde-crosslinked PU75 nanofibers showed flattened and fused fibers, indicative of partial dissolution.

Engineered vascular scaffold features, such as porosity, mean pore diameter, and surface hydrophilicity, are critical for tissue regeneration or repair processes. ${ }^{27}$ Among the multitudinous factors that affect the hydrophilicity of scaffold, the chemical structure of the materials is a crucial one. Scaffolds which possess a surface with carboxyl, amino, or hydroxy groups will show hydrophilic characteristics (the water contact angle is less than $\left.90^{\circ}\right) \cdot{ }^{11}$ As in our previous studies, ${ }^{17}$ the pure PEUU nanofibrous membrane tends to be hydrophobic, with a steady water contact angle of $120^{\circ}$. The water droplet cannot spread well on the surface of the hydrophobic, pure PEUU nanofibrous membrane, which is a disadvantage for cell spreading and proliferation. It can be seen from Table 1 that the contact angles of PU75 membranes decrease after PEUU is blended with gelatin due to the gelatin's hydrophilicity. When comparing the PU75 and PU75-DT nanofibrous membranes, there is only a slight increase of contact angle after dehydrothermal treatment. This could be the reason that the porosity and mean pore diameter of the PU75-DT is less than the PU75 nanofibrous membrane. It can also be concluded from Table 1 that the contact angles of the nanofibrous membranes increase after being crosslinked with glutaraldehyde vapor and EDC/NHS for 5 seconds, when compared with the PU75 nanofibrous membrane. This is likely due to the increase in the diameter nanofibrous material after dehydrothermal and EDC/ NHS solution treatment, thus shrinking the pore diameter (Table 1) and making the water flow down to the lower side along the larger pore tunnel. Moreover, the water contact angle of the PU75-GA nanofibrous membrane is smaller than the PU75-DT and PU75-E/N nanofibrous membranes, primarily due to the redundant carboxyl group on the PU75-GA molecular chain, as shown in Fig. 2.

\subsection{Chemical structures of nanofibers}

The successful crosslinking of PU75 was qualitatively confirmed by ATR-FTIR spectroscopy (Fig. 3(a)). The main chain of PEUU contains masses of $\mathrm{CH}_{2}$ sections, present in both the hard and the soft segments. The structure of the sections containing $\mathrm{CH}_{2}$ are identified by the peaks at $2937 \mathrm{~cm}^{-1}$ and $2863 \mathrm{~cm}^{-1}$, which represent the stretching vibrations of $\mathrm{H}-\mathrm{C}-\mathrm{H}$. The strong peak at $1730 \mathrm{~cm}^{-1}$ represents the stretching vibration of $\mathrm{C}=\mathrm{O}$, the peak at $1570 \mathrm{~cm}^{-1}$ represents the $\mathrm{N}-\mathrm{H}$ deformation vibration, and the broad peaks at $1161 \mathrm{~cm}^{-1}$ to $1236 \mathrm{~cm}^{-1}$ represent the stretching vibration of C-O-C. ${ }^{15,17}$ The broad peak at 3500$3300 \mathrm{~cm}^{-1}$ represents the stretching vibration of $\mathrm{O}-\mathrm{H}$, which exists abundantly in the gelatin molecular chain. The gelatin's 

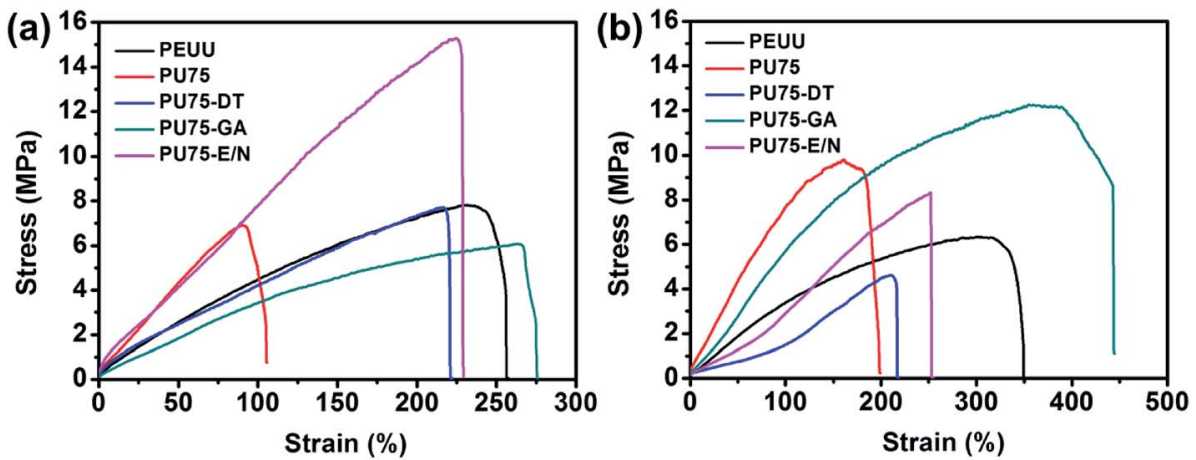

Fig. 4 Typical stress-strain curves of the PEUU nanofibrous membrane, PU75 nanofibrous membrane, PU75-DT nanofibrous membrane, PU75GA nanofibrous membrane, and PU75-E/N nanofibrous membrane in dry states (a) and wet states (b).

intense peak at $1570 \mathrm{~cm}^{-1}$ may be due to the higher density of the $\mathrm{N}-\mathrm{H}$ molecular structure. The peak's magnitude does not change significantly when crosslinking treatment after gelatin was blended in PEUU, might due to the a few additions of gelatin in the PU75 nanofibers. The absorption peaks highly overlap with those of the amide and ester groups in PEUU and gelatin, as shown in Fig. 2. The crystalline structural analysis of gelatin and PU75 nanofibers both before and after crosslinking was explored, as shown in Fig. 3(b), where crystalline zones display sharp diffraction peaks and amorphous regions show

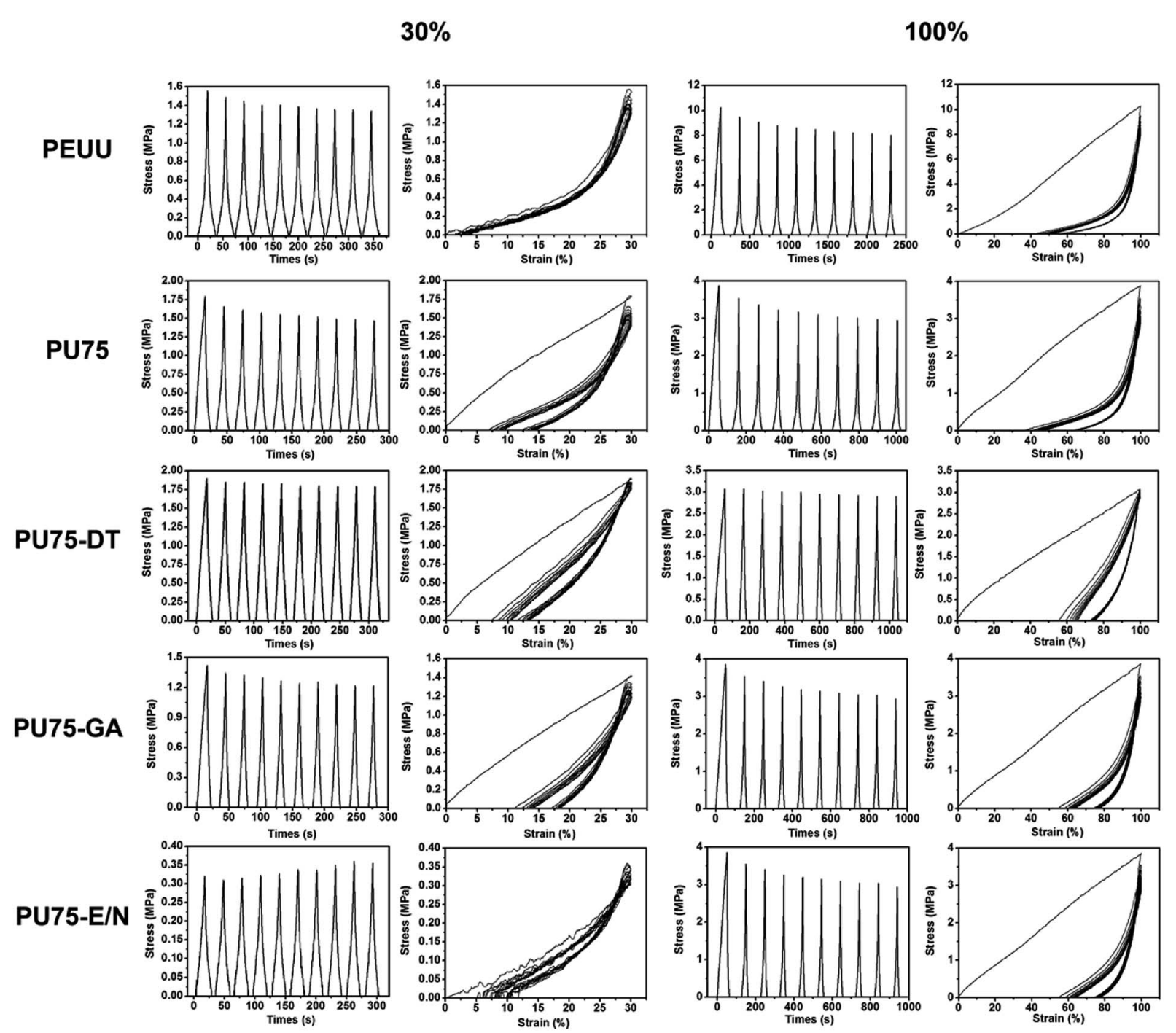

Fig. 5 Cyclic tensile response curves at 30\% deformation and 100\% deformation of the PEUU nanofibrous membrane, PU75 nanofibrous membrane, PU75-DT nanofibrous membrane, PU75-GA nanofibrous membrane, and PU75-E/N nanofibrous membrane in dry states. 
(a)
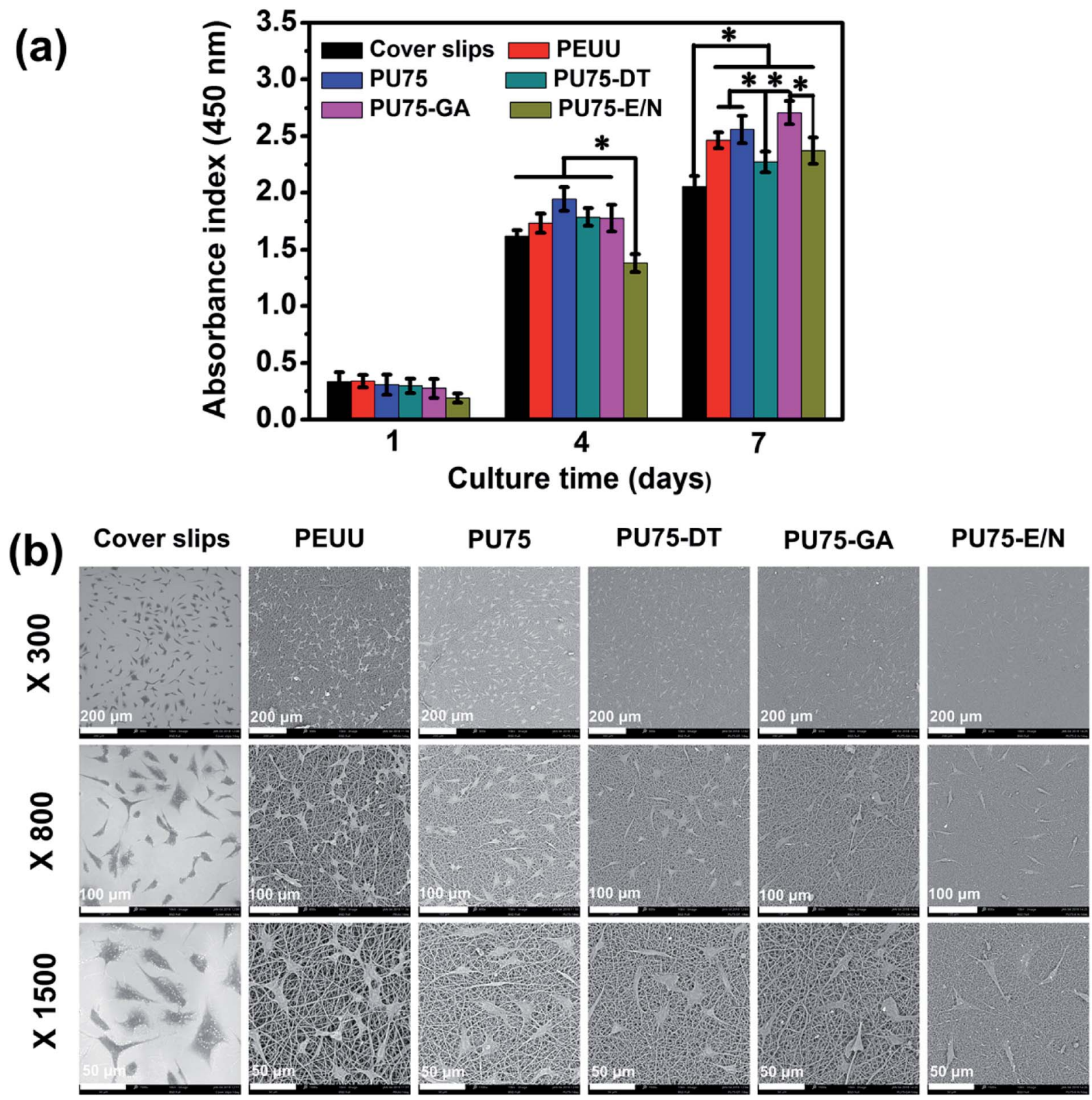

Fig. 6 (a) CCK-8 assay of the proliferation viability and (b) SEM micrographs after a 4 day culture of L929 cells on coverslips, PEUU nanofibrous membranes, PU75 nanofibrous membranes, PU75-DT nanofibrous membranes, PU75-GA nanofibrous membranes, and PU75-E/N nanofibrous membranes, magnified 300 times, 800 times, and 1500 times.

broader ones. The absence of a diffraction peak in the WAXRD pattern of the gelatin shows that the gelatin is amorphous. The PEUU polymer had one different peak at $2 \theta$ around $21.5^{\circ} .^{15,16}$ The gelatin, a hydrolyzed collagen, has some inherited crystalline segments of collagen, particularly at about $20.4^{\circ}$. However, the orientation of the gelatin and the PEUU molecular chains during the electrospinning process also affects the PEUU nanofiber crystallization. The WAXRD pattern of the PEUU nanofibers shows that the crystallization of PEUU nanofibers is diffuse. Moreover, it is obvious that the curves of PEUU and PU75 are analogous. This is because there is no obvious molecular chain glide during the hybridization process. In addition, the crystal alignment was caused by the molecular- chain-moving-effect during crosslinking treatment, which is especially prominent in the dehydrothermal-treated PU75 nanofibers.

\subsection{Mechanical properties of nanofibrous scaffolds}

Vascular scaffolds have strict mechanical property requirements to mimic native blood vessels and to fulfill surgical needs. ${ }^{28}$ Various tests were performed on PEUU, PU75, PU75DT, PU75-GA, and PU75-E/N nanofibrous scaffolds to evaluate their mechanical properties, as shown in Table 2, Fig. 4, and Fig. 5. Fig. 4(a) shows the representative stress-strain curves in dry states, from which it was found that the crosslinked 


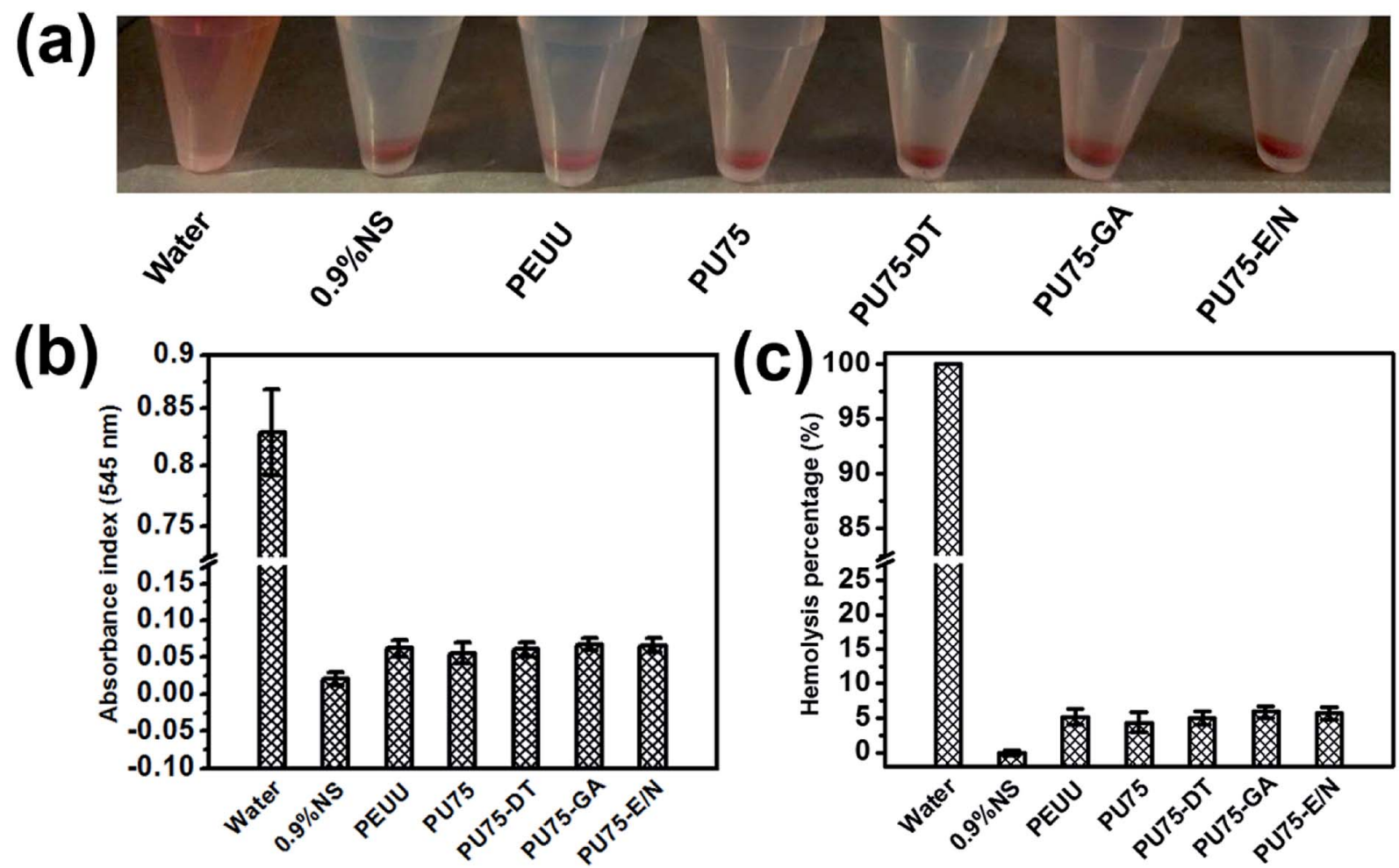

Fig. 7 (a) Photo of a solution of human red blood cells (HRBCs) treated with different nanofibrous samples, followed by centrifugation, (b) the absorbance at $545 \mathrm{~nm}$ of the supernatant (hemoglobin) and (c) relative hemolysis of HRBCs after being incubated with water, $0.9 \%$ NS, PEUU nanofibrous membrane, PU75 nanofibrous membrane, PU75-DT nanofibrous membrane, PU75-GA nanofibrous membrane, and PU75-E/N nanofibrous membrane, respectively.

nanofibrous membranes showed greater strain than the PU75 nanofibrous membranes. In addition, PU75-E/N nanofibrous membranes can withstand far greater stress than other specimens. This is due to the huge amide bond network in PU75-E/N nanofibers after the EDC/NHS solution crosslinking treatment. Table 2 shows the Young's modulus results, revealing that the PU75 nanofibrous membranes had the highest Young's modulus, whereas those membranes with crosslinking treatment had relatively low values. Overall, the Young's modulus of all of the membranes was higher than that of porcine coronary arteries (about $1 \mathrm{MPa}$ ). Fig. 4(b) shows the representative stressstrain curves in wet states, from which it was found that the PU75-DT nanofibrous membrane and PU75-E/N nanofibrous membrane showed similar strain-tolerance in both dry and wet states. This is due to their lower porosity. Additionally, the tensile strengths of the PU75 nanofibrous membranes and PU75-GA nanofibrous membranes in the wet state are slightly higher than their corresponding values in the dry state. As seen in Table 1, the water contact angle of the PU75 and the PU75-GA nanofibrous membranes are less than the PU75-DT and PU75-E/ $\mathrm{N}$ nanofibrous membranes. For the PU75 nanofibrous membranes, after soaking the membrane in water, some gelatin was dissolved from PU75, causing the PEUU molecule chains to rearrange and resulting in recrystallization and an increased tensile strength. Furthermore, water could be absorbed inside the porous structure in the nanofibers, replacing the air gaps. The water may act as an inter-fiber bridge to form a contact region, which increases the interaction between physically stacking nanofibers, resulting in a more heterogeneous stress distribution. As a result, the tensile strength of the membranes in the wet state increased. ${ }^{24,29}$ The other two important mechanical variables to consider are the suture retention strength and the burst pressure strength, both shown in Table 2. The suture retention strengths of the PEUU nanofibrous scaffolds were lower than the other specimens' values, which is attributed to the hybrid gelatin improving the stress of the composite nanofibers. The burst pressure results showed that the PU75 nanofibrous scaffolds had lower values than the other specimens due to the brittleness of gelatin. All the above mechanical properties of the PU75-GA nanofibrous scaffold surpassed porcine coronary artery values (tensile strength $2.8 \mathrm{MPa}$, elongation at break $120 \%$ ), human saphenous vein (suture retention strength $1.9 \mathrm{~N}$, burst pressure $3000 \mathrm{mmHg}$ ), human carotid artery (burst pressure $5800 \mathrm{mmHg}$ ), and human mammary artery (suture retention strength $1.2 \mathrm{~N}$ ). ${ }^{28}$

The cyclical mechanical properties reflect the sustainability of these nanofibrous scaffolds in the long term. ${ }^{12,30,31}$ The cyclical tensile test results are shown in Fig. 5. Cyclic tensile loading was performed with a maximum strain of either $30 \%$ or $100 \%$. The stretch and release tests were repeated 10 times, with the area enclosed in the stretch and release curves at each time point representing the energy loss for each cycle. It was found that most of the specimens exhibited a large hysteresis loop in the first cycle, followed by much smaller hysteresis loops in the next 9 cycles, with the exception of the PEUU nanofibrous membrane. At a low deformation of $30 \%$, the PU75, PU75-DT, 
(a)
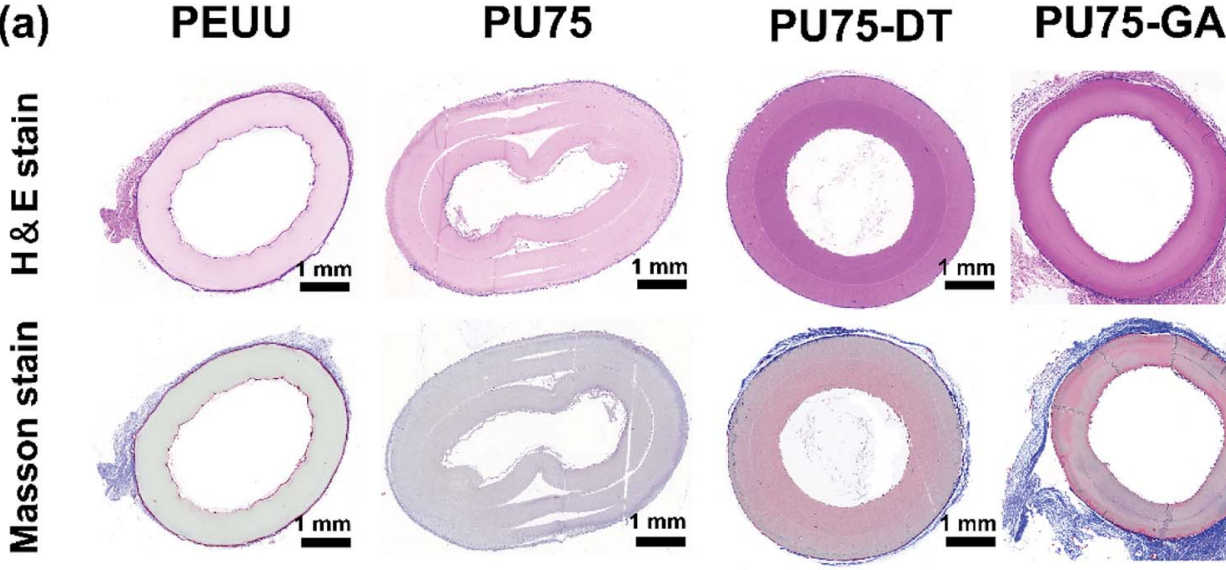

PU75-E/N

(b)
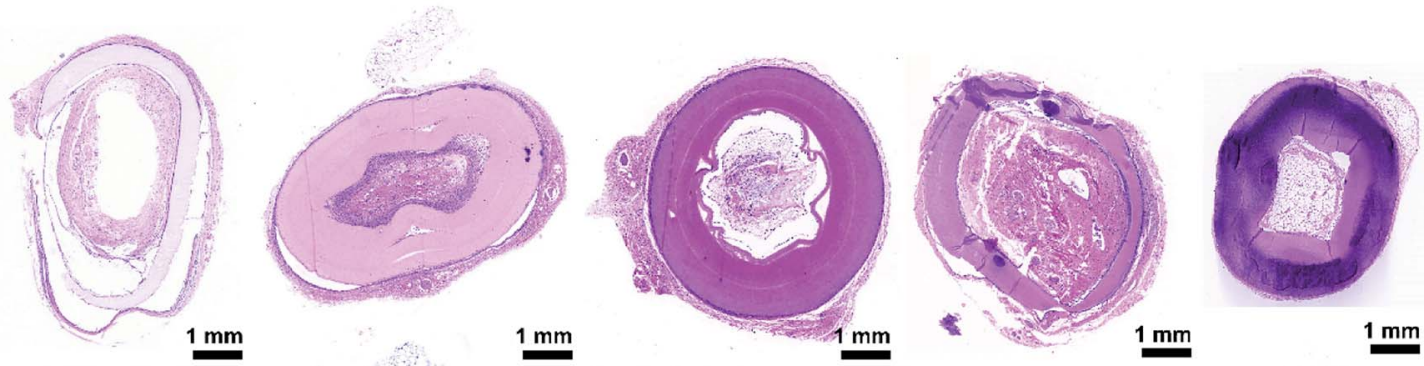

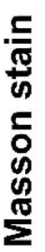
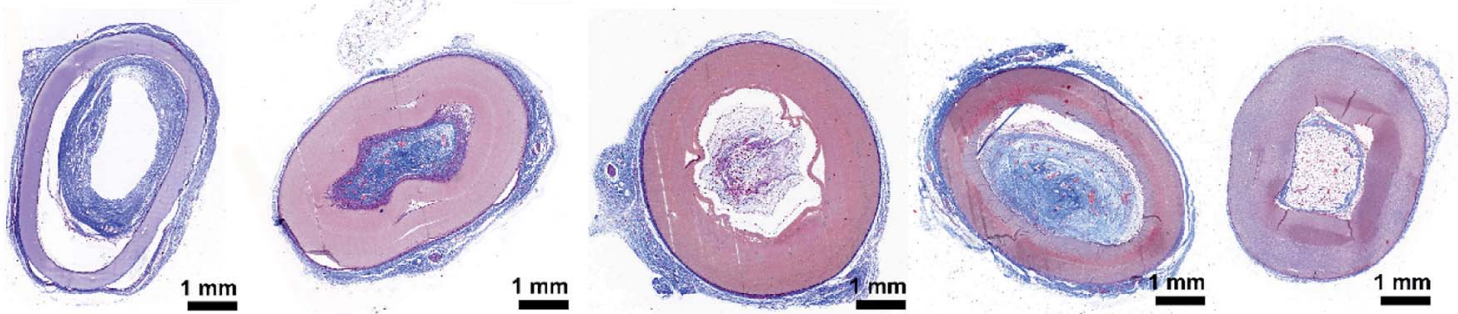

Fig. 8 H\&E staining images and Masson's trichrome staining images of the transplanted PEUU nanofibrous tubular scaffold, PU75 nanofibrous tubular scaffold, PU75-DT nanofibrous tubular scaffold, PU75-GA nanofibrous tubular scaffold, and PU75-E/N nanofibrous tubular scaffold after subcutaneous embedding in mice for 4 weeks (a) and 8 weeks (b).

PU75-GA, and PU75-E/N nanofibrous membranes showed a small unrecoverable deformation (about 10\%) due to the fragility of gelatin, which is unable to regain its original shape after having been stretched. At large strains of $100 \%$, the unrecoverable deformations became much more appreciable (about 40\%) for the PEUU, PU75, PU75-DT, and PU75-GA nanofibrous membranes, while the PU75-E/N nanofibrous membrane values were about $80 \%$. It was decided that a disrupted crystallinity in polymers would lead to less large-straininduced crystallization and more strain recovery. These results are consistent with a previous report by Fang et al. ${ }^{\mathbf{1 4}}$

\subsection{Cell viability and morphology}

CCK-8 assays detecting absorbance at $450 \mathrm{~nm}$ on days 1,4 , and 7 were utilized to evaluate the proliferation of L929 cells grown on the nanofibrous membranes in vitro. As shown in Fig. 6(a), L929 cells grown on the nanofibrous membrane proliferated faster than those on cover slips during the long culture period (7 days), suggesting excellent biocompatibility. The viability of L929 cells after a 4 day culture on PU75-E/N nanofibrous membranes is significantly lower than other groups, possibly due to the existence of residual EDC/NHS, which provides an adverse alkaline environment for cell growth. The cell proliferation rate of PU75-GA nanofibrous membranes on the $7^{\text {th }}$ day was dramatically larger than the other samples. This implies that the hydrophilic properties of the materials were a decisive factor for cell growth.

The morphologies of the L929 cells grown for 4 days on coverslips, and on PEUU, PU75, PU75-DT, PU75-GA, and PU75$\mathrm{E} / \mathrm{N}$ nanofibrous membranes were observed via SEM. As shown in Fig. 6(b), it can be seen that the cell shapes of the nanofibrous membrane groups tend to be multi-directional, rather than round, with the L929 cells cultured on the nanofibrous membrane presenting a much better morphology than those on coverslips. Compared to the PU75-E/N nanofibrous membranes group, the amounts of fusiform cells on the PEUU nanofibrous membrane, PU75 nanofibrous membrane, PU75DT nanofibrous membranes, and PU75-GA nanofibrous membranes were markedly greater than the PU75-E/N nanofibrous membrane group. Moreover, cells on the PEUU and PU75 nanofibrous membranes tend to be more stretchable than the crosslinked nanofibrous membranes groups. The cell 
morphology results were consistent with the cell proliferation results. Here, the cell viability and morphology results indicate that nanofiber features can significantly influence the proliferation of cells, though the crosslinking process need to be further controlled.

\subsection{Hemocompatibility of nanofibrous scaffolds}

The hemocompatibility of biomaterials is an important factor in vascular tissue engineering applications. The presence of certain materials can negatively affect red blood cells and destroy their cell membranes, causing the release of hemoglobin into solution. Hemolysis rates were measured using a colorimetric assay of released hemoglobin from red blood cells. As shown in Fig. 7, the hemolysis test results of the PEUU, PU75, PU75-DT, PU75-GA, and PU75-E/N nanofibrous membranes are all less than the safe limit value (5\%). PU75 nanofibrous membranes have a lower hemolysis rate than that of the crosslinking nanofibrous membranes, possibly due to the existence of residual agents. In general, these results indicate that PEUU, PU75, and crosslinking PU75 nanofibrous membranes exhibit reliable blood biocompatibility and may be developed as a substitute for artificial blood vessels in clinical usage.

\subsection{Histological analysis after subcutaneous implantation}

Subcutaneous implantation (implanted in the backs of 6 weekold male mice) was used to assess the in vivo biocompatibility of the crosslinked nanofibrous scaffolds and PEUU nanofibrous scaffold, with the PU75 nanofibrous scaffold used as a control. Materials with different chemical surfaces may lead to different inflammatory responses in the tissues of mice. Histological analysis of transverse sections at the middle sites were performed by staining with hematoxylin and eosin (H\&E), and Masson's trichrome to observe the inflammatory response. ${ }^{26,32,33}$ During the experiment, all mice remained in good health, with no wound complications. For all nanofibrous tubular scaffolds, no acute or chronic inflammation, necrosis, or adverse tissue reactions were identified. ${ }^{29}$ Fig. 8 shows that the tissues from all specimens present no gross inflammation. At 4 weeks, all the nanofibrous scaffolds maintained their integral structure, with the exception of the PU75 nanofibrous scaffold. The host cells proliferated on the outside surface of the nanofibrous scaffolds, as shown in the images of H\&E staining. The sections of PU75 nanofibrous tubular scaffold implanted subcutaneously collapsed after 4 weeks, possibly due to the dissolution of gelatin in the PU75 nanofibers. The Masson's trichrome staining suggested the production of collagen fibers secreted by the infiltrated cells on the outer layer surface of nanofibrous scaffolds. It indicated that infiltrated cells preserved the functionality, with the produced collagen fibers providing a biomimetic environment for tissue regeneration. After 8 weeks of postsubcutaneous implantation, the autologous tissue had gone up into the lumen. A mass of fibroblasts and blood vessels are observed in the inner part of the PU75-GA nanofibrous tubes, most of which are being replaced by a fibrous tissue with part of the walls disappearing. The integration of the membrane and tissue at the interface was thus achieved. ${ }^{26}$ All the results demonstrated that suitable pore size and porosity, along with excellent mechanical support, are the critical factors for tissue construction.

\section{Conclusions}

In this study, PEUU/gelatin composites, with weight ratios of 75/ 25 , nanofibrous membranes, and nanofibrous tubular scaffolds were produced via electrospinning technology and then crosslinked using different techniques. It was found that the PU75GA nanofibrous scaffold exhibited a combination of proper pore diameter, high biocompatibility, and reliable blood biocompatibility. In addition, not only did the crosslinking of PU75 nanofibers create obvious changes in the hydrophilicity of the materials, but it also improved their mechanical properties. Furthermore, the pore structure of fibers may be improved using different crosslinking technologies. Therefore, developing the technology to combine electrospinning with glutaraldehyde vapor crosslinking will provide excellent mechanical properties, high porosity, and pore interconnectivity scaffolding for facilitating cell penetration.

\section{Ethical statement}

All animal procedures were performed in accordance with the Guidelines for Care and Use of Laboratory Animals of Fudan University and approved by the Animal Ethics Committee of Zhongshan Hospital.

\section{Conflicts of interest}

There are no conflicts to declare.

\section{Acknowledgements}

The authors sincerely appreciate the supports of "National Major Research Program of China (Grant No. 2016YFC1100200)", "National Nature Science Foundation (Grant No. 81501595, 31470941, 31771023)", "Science and Technology Commission of Shanghai Municipality (Grant No. 15JC1490100, 15441905100)", "Yantai Double Hundred Talent Plan", and "light of textile project (Grant No. J201404)"; "Youth Foundation of Zhongshan Hospital (Grant No. 2015ZSQN09)", "Talent Training Program Foundation for the Excellent Youth Supported by Zhongshan Hospital (Grant No. 2017ZSYQ24)", and "Innovation Fund of Zhongshan Hospital (Grant No. 2017ZSCX05)”.

\section{References}

1 U. Sharma, D. Concagh, L. Core, Y. Kuang, C. C. You, Q. Pham, G. Zugates, R. Busold, S. Webber, J. Merlo, R. Langer, G. M. Whitesides and M. Palasis, Nat. Mater., 2018, 17, 96-103.

2 G. C. Ingavle and J. K. Leach, Tissue Eng., Part B, 2014, 20, 277-293. 
3 S. H. Chen, Y. Chang, K. R. Lee and J.-Y. Lai, J. Membr. Sci., 2014, 450, 224-234.

4 J. R. Dias, S. Baptista-Silva, C. M. T. de Oliveira, A. Sousa, A. L. Oliveira, P. J. Bártolo and P. L. Granja, Eur. Polym. J., 2017, 95, 161-173.

5 Y. C. Jiang, L. Jiang, A. Huang, X. F. Wang, Q. Li and L. S. Turng, Mater. Sci. Eng., C, 2017, 71, 901-908.

6 S. J. Liu, Y. C. Kau, C. Y. Chou, J. K. Chen, R. C. Wu and W. L. Yeh, J. Membr. Sci., 2010, 355, 53-59.

7 H. A. Strobel, E. L. Calamari, A. Beliveau, A. Jain and M. W. Rolle, J. Biomed. Mater. Res., Part A, 2018, 106, 817-826.

8 N. Bhardwaj and S. C. Kundu, Biotechnol. Adv., 2010, 28, 325347.

9 Z. M. Du, N. F. Li, Y. J. Hua, Y. Shi, C. Y. Bao, H. B. Zhang, Y. Yang, Q. N. Lin and L. Y. Zhu, Chem. Commun., 2017, 53, 13023-13026.

10 C. C. Xu, Y. H. Huang, L. P. Tang and Y. Hong, ACS Appl. Mater. Interfaces, 2017, 9, 2169-2180.

11 J. J. Guan, M. S. Sacks, E. J. Beckman and W. R. Wanger, J. Biomed. Mater. Res., Part A, 2002, 61, 493-503.

12 J. Fang, S. H. Ye, J. Wang, T. Zhao, X. M. Mo and W. R. Wanger, Biomacromolecules, 2015, 16, 1622-1633.

13 Y. Hong, A. Huber, K. Takanari, N. J. Amoroso, R. Hashizume, S. F. Badylak and W. R. Wanger, Biomaterials, 2011, 32, 3387-3394.

14 J. Fang, S.-H. Ye, V. Shankarraman, Y. X. Huang, X. M. Mo and W. R. Wanger, Acta Biomater., 2014, 10, 4639-4649.

15 K. Yu, X. X. Zhou, T. H. Zhu, T. Wu, J. Wang, J. Fang, M. R. ElAssar, H. El-Hamshary, M. El-Newehy and X. M. Mo, RSC Adv., 2016, 6, 73636-73644.

16 H. R. Bakhsheshi-Rad, Z. Hadisi, E. Hamzah, A. F. Ismail, M. Aziz and M. Kashefian, Mater. Lett., 2017, 207, 179-182.

17 T. H. Zhu, K. Yu, M. A. Bhutto, X. R. Guo, W. Shen, J. Wang, W. M. Chen, H. El-Hamshary, S. S. Al-Deyab and X. M. Mo, Chem. Eng. J., 2017, 315, 177-190.

18 K. Yu, T. H. Zhu, Y. Wu, X. X. Zhou, X. X. Yang, J. Wang, J. Fang, H. El-Hamshary, S. S. Al-Deyab and X. M. Mo, Colloids Surf., B, 2017, 151, 314-323.

19 A. Laha, C. S. Sharma and S. Majumdar, Mater. Sci. Eng., C, 2017, 76, 782-786.
20 S. R. Goes, G. Rodrigues, G. G. Martins, C. M. R. Henriques and J. C. Silva, Mater. Sci. Eng., C, 2013, 33, 1219-1227.

21 A. P. Kishan, R. M. Nezarati, C. M. Radzicki, A. L. Renfro, J. L. Robinson, M. E. Whitely and E. M. CosgriffHernandez, J. Mater. Chem. B, 2015, 3, 7930-7938.

22 W. M. Chen, S. Chen, Y. Morsi, H. El-Hamshary, M. ElNewhy, C. Y. Fan and X. M. Mo, ACS Appl. Mater. Interfaces, 2016, 8, 24415-24425.

23 X. X. Yang, X. Y. Wang, F. Yu, L. L. Ma, X. H. Pan, G. J. Luo, S. Lin, X. M. Mo, C. L. He and H. S. Wang, RSC Adv., 2016, 6, 99720-99728.

24 E. S. Jamadi, L. Ghasemi-Mobarakeh, M. Morshed, M. Sadeghi, M. P. Prabhakaran and S. Ramakrishna, Mater. Sci. Eng., C, 2016, 63, 106-116.

25 T. Wu, C. Huang, D. W. Li, A. L. Yin, W. Liu, J. Wang, J. F. Chen, H. Ei-Hamshary, S. S. Al-Deyab and X. M. Mo, Colloids Surf., B, 2015, 133, 179-188.

26 J. J. Xue, M. He, Y. Z. Liang, A. Crawford, P. Coates, D. F. Chen, R. Shi and L. Q. Zhang, J. Mater. Chem. B, 2014, 2, 6867-6877.

27 J. L. Wu, C. Huang, W. Liu, A. L. Yin, W. M. Chen, C. L. He, H. S. Wang, S. Liu, C. Y. Fan, G. L. Bowlin and X. M. Mo, J. Biomed. Nanotechnol., 2014, 10, 603-614.

28 H. Y. Mi, X. Jing, E. Yu, J. McNulty, M. R. Salick, X. F. Peng and L. S. Turng, Ind. Eng. Chem. Res., 2016, 55, 882-892.

29 R. Shi, J. J. Xue, H. B. Wang, R. X. Wang, M. Gong, D. F. Chen, L. Q. Zhang and W. Tian, J. Mater. Chem. B, 2015, 3, 40634073.

30 T. Wu, J. L. Zhang, Y. F. Wang, D. D. Li, B. B. Sun, H. ElHamshary, M. Yin and X. M. Mo, Mater. Sci. Eng., C, 2018, 82, 121-129.

31 Y. Si, J. Yu, X. Tang, J. Ge and B. Ding, Nat. Commun., 2014, 5, 5802.

32 W. Z. Wang, J. W. Hu, C. L. He, W. Nie, W. Feng, K. X. Qiu, X. J. Zhou, Y. Gao and G. Q. Wang, J. Biomed. Mater. Res., Part A, 2015, 103, 1784-1797.

33 Y. T. Jia, W. Z. WanG, X. J. Zhou, W. Nie, L. Chen and C. L. He, Polym. Chem., 2016, 7, 2553-2564. 